\title{
SOME REMARKS ON THE STABILITY OF A PROPERTY RELATED TO THE MEAN VALUE THEOREM FOR HARMONIC FUNCTIONS
}

\author{
BURTON RANDOL
}

(Communicated by Clifford J. Earle, Jr.)

\begin{abstract}
Suppose $u$ is harmonic and of mean zero over a compact domain $D$. We study the extent to which the zero-set of $u$ must penetrate into the interior of $D$.
\end{abstract}

Suppose $u(z)(z=x+i y)$ is smooth on the closed unit disk $\Delta$ and harmonic in its interior. By the area form of the mean value theorem,

$$
u(0)=\frac{1}{\pi} \iint_{\Delta} u d x d y .
$$

In particular, if it is the case that the average of $u$ over $\Delta$ is zero, then (1) tells us that $u(0)=0$, one consequence of which is that, for such functions, the zero-set $V(u)$ of $u$ always penetrates a definite distance (in this case unit distance) into the interior of $\Delta$, independently of $u$.

Recently, Lisa Goldberg asked, in connection with questions in Teichmüller theory, whether or not this last property is special to the disk, or is in fact shared by fairly general sets. In this note, we will address some aspects of this question, and show that the answers are surprisingly delicate.

In more detail, suppose that $D$ is a simply connected compact domain having smooth boundary $\partial D$, and that $u$ is $C^{\infty}$ on $D$ and harmonic in its interior, and satisfies $\iint_{D} u d x d y=0$. Is it then the case that there exists an $\epsilon=\epsilon(D)>$ 0 such that $V(u)$ penetrates at least $\epsilon$ units into $D$ ? I.e., is there always a point $z_{0} \in V(u)$ whose distance from $\partial D$ is greater than or equal to $\epsilon$ ? If this is the case, we will say that $D$ has Property $\mathrm{P}$. Note that Property $\mathrm{P}$ is true in the presence of a general mean value theorem, i.e., if there exists a point $z_{1}$ in the interior of $D$ such that $u\left(z_{1}\right)=|D|^{-1} \iint_{D} u d x d y$ for all harmonic $u$, where $|D|=\operatorname{area}(D)$. It is well known, however, that the existence of such a point characterizes a disk [3], so this approach cannot suffice in a general context. More generally, Property $P$ would be established if there is associated with $D$ a compact subset $C$ of the interior of $D$, and a nonnegative Borel measure $d \mu$ supported on $C$, such that $\iint_{D} u d x d y=\iint_{C} u d \mu$ for each $u$. This is known to occur for some domains. For example, if $D$ is an ellipse, the

Received by the editors July 2, 1990.

1980 Mathematics Subject Classification (1985 Revision). Primary 30C15, 31A05, 31 B05. 
segment connecting the two foci is such a $C$, for which the measure $d \mu$ has a simple explicit description $[1,2,5]$. On the other hand, Property $\mathrm{P}$ is apparently weaker than a quadrature property of this kind (but cf. the remark at the end of this note).

We next note that Property $\mathrm{P}$ would be established for a domain $D$ if we could establish the existence of a positive lower bound, depending only on $D$, for the area occupied by the subset of $D$ upon which $u$ is positive. As it happens, such a lower bound does not even exist for the disk. One quite explicit way of seeing this makes use of an entire function $E_{\alpha}(z)$, introduced by MittagLeffler [4], and defined, for $\alpha>0$, by

$$
E_{\alpha}(z)=\sum_{n=0}^{\infty} \frac{z^{n}}{\Gamma(n \alpha+1)} .
$$

It was established in [4] that $E_{\alpha}(z)$ tends uniformly to zero as $z \rightarrow \infty$ in the complement of any cone having vertex at the origin, symmetry about the positive real axis, and angular opening greater than $\pi \alpha$. (Note that $E_{\alpha}(0)=1$.)

Now, for fixed small $\alpha>0$, define a family $F_{1}(z), F_{2}(z), \ldots$ of entire functions by setting $F_{n}(z)=E_{\alpha}(n z)$, and a family of real harmonic functions $u_{1}(z), u_{2}(z), \ldots$, by setting $u_{n}(z)=\operatorname{Re}\left(F_{n}(z)-1\right)$.

If we consider the $u_{n}$ 's as a family of harmonic functions in $\Delta$, it is then evident that

(1) $\iint_{\Delta} u_{n}=0(n=0,1, \ldots)$, since $u_{n}(0)=0$.

(2) $u_{n} \rightarrow-1$ uniformly in the complement within $\Delta$ of the union of any fixed neighborhood of the origin with a cone of the above type.

It thus immediately follows that there are $u_{n}$ 's for which the area of the set of positivity is arbitrarily small.

We will next show that the disk is unstable with respect to Property $P$, even within the space of $C^{\infty}$ deformations.

For this purpose, consider a function $\varphi$ of the form

$$
z+a_{2} z^{2}+a_{3} z^{3}, \ldots
$$

for which the $a_{n}$ 's are nonnegative $\left(a_{1}=1\right)$, and chosen so that

(1) $\varphi(z)$ is holomorphic in $|z|<1$ and $C^{\infty}$ on $|z|=1$.

(2) $a_{n}=\Omega\left(\beta^{n}\right)$ for any $\beta<1$.

(3) $\varphi(z)$ is a homeomorphism on $|z| \leq 1$.

These requirements will be satisfied, for example, if $a_{n}=0$ for $2 \leq n \leq N$ for a sufficiently large $N$, while $a_{n}=n^{-[\log n]}$ for $n>N$.

Now consider, for a large integer $M$, the function $f$ on $D=\varphi(\Delta)$ given by $f(z)=\left(\varphi^{-1}(z)\right)^{M-1}$.

Note that for any $\epsilon>0$, there exists $\delta<1$ such that for points $z$ of $D$ at distance $\geq \epsilon$ from the boundary of $D,|f(z)| \leq \delta^{M}$, for sufficiently large $M$.

Choose $\beta \in(\delta, 1)$, and compute $\iint_{D} f d x d y=\iint_{\Delta} z^{M-1}\left|\varphi^{\prime}(z)\right|^{2} d x d y$.

Now

$$
\varphi^{\prime}(z)=\sum_{n=1}^{\infty} n a_{n} z^{n-1},
$$


so that

$$
\left|\varphi^{\prime}(z)\right|^{2}=\sum_{n=1}^{\infty} \sum_{m=1}^{\infty} m n a_{m} a_{n} z^{m-1} \bar{z}^{n-1}
$$

or

$$
\iint_{\Delta} z^{M-1}\left|\varphi^{\prime}(z)\right|^{2} d x d y=\iint_{\Delta} \sum_{n=1}^{\infty} \sum_{m=1}^{\infty} m n a_{m} a_{n} z^{M+m-2} \bar{z}^{n-1} d x d y,
$$

which in polar coordinates becomes

$$
\begin{gathered}
\int_{0}^{2 \pi} d \theta \int_{0}^{1}\left(\sum_{n=1}^{\infty} \sum_{m=1}^{\infty} m n a_{m} a_{n} r^{M+m+n-2} e^{i(M+m-n-1) \theta}\right) d r \\
=\int_{0}^{2 \pi}\left(\sum_{n=1}^{\infty} \sum_{m=1}^{\infty} \frac{m n}{M+m+n-1} a_{m} a_{n} e^{i(M+m-n-1) \theta}\right) d \theta \\
=\pi \sum_{m=1}^{\infty} m a_{m} a_{M+m-1} \geq \pi a_{M}=\Omega\left(\beta^{M}\right) .
\end{gathered}
$$

I.e., $A_{M}=|D|^{-1} \iint_{D} f d x d y=\Omega\left(\beta^{M}\right)$. Now $\delta^{M}$ tends to zero much more rapidly than $\beta^{M}$, so if we set $u_{M}=\operatorname{Re}\left(f(z)-A_{M}\right)$, it follows from our previous remarks that by taking $M$ large, $V\left(u_{M}\right)$ can be made to penetrate into $D$ by as little as we wish, which establishes the failure of Property $\mathrm{P}$ for $D$. Note that this example also shows that the integral of a harmonic function over such a $D$ cannot be universally obtained by integration over a finite Borel measure whose effect is computable by restriction to the interior of $D$.

We conclude with a result in the positive direction, for which we will need the following definition (Property Q). We will show that under certain circumstances, the analyticity of $\partial D$ implies that $D$ has Property $\mathrm{Q}$. What follows should be regarded as the illustration of a method in an interesting case, rather than an attempt to formulate the most general conditions under which the desired conclusions are valid.

Definition. Suppose $u$ is harmonic on $D$ and $\iint_{D} u d x d y=0$. Then $u$ has a conjugate $v$, which we also normalize by requiring that $\iint_{D} v d x d y=0$. If there exists an $\epsilon=\epsilon(D)>0$ such that either $V(u)$ or $V(v)$ always penetrates at least $\epsilon$ units into $D$, we will say that $D$ has Property Q.

Theorem. Suppose $\partial D$ is analytic, and is sufficiently $C^{2}$-close to $\partial \Delta$. I.e., there is a $C^{2}$ homeomorphism between $\partial D$ and $\partial \Delta$ that is $C^{2}$-close to the identity. Then $D$ has Property Q.

Proof. Let $u$ and $v$ be as above, and consider the function $f=u+i v$, which is holomorphic in $D$, and satisfies $\iint_{D} f d x d y=0$.

Now it follows from a complex form of Green's theorem [2, p. 125], that

$$
\iint_{D} f d x d y=\frac{1}{2 i} \int_{\partial D} \bar{z} f(z) d z
$$

Furthermore, since $\partial D$ is analytic, it can be shown that $\bar{z}$ is the restriction to $\partial D$ of a holomorphic function $S(z)$, the so-called Schwarz function, defined in some neighborhood of $\partial D$ (cf. [2]). I.e., 


$$
\iint_{D} f d x d y=\frac{1}{2 i} \int_{\partial D} S(z) f(z) d z
$$

Cauchy's theorem therefore permits us to replace $\partial D$ in the last integral by a nearby curve $\gamma$ lying in the interior of $D$, so that

$$
2 i \iint_{D} f d x d y=\int_{\gamma} S(z) f(z) d z=0 .
$$

(Note for later use that we may assume, by taking $\gamma$ close to $\partial D$, that $S$ does not vanish in a neighborhood of $\partial D$ that includes $\gamma$, and that $f$ does not vanish on $\gamma$. This follows from the observations that on $\partial D, S(z)=\bar{z}$, which is not zero if $\partial D$ is near the unit circle, while if $f$ vanished on $\gamma$, the desired result would be established for both $u$ and $v$, and we would be done.)

The last equality implies that there exists a well-defined antiderivative $F(z)$ of $S(z) f(z)$ in a neighborhood of $\gamma$. I.e., $S(z) f(z)=F^{\prime}(z)$.

Now let $\Gamma$ be the image of $\gamma$ under the map $F$, and consider the relationship of the unit tangent fields along $\gamma$ and $\Gamma$, where we are tracing $\gamma$ in the counterclockwise direction. As we complete a circuit around $\gamma$, so that the unit tangent vector rotates through $2 \pi$ radians (since $\gamma$ is close to the unit circle), it is straightforward to see (by considering $(F(z+\Delta z)-F(z)) / \Delta z$, where $z$ and $z+\Delta z$ lie on $\gamma$ ), that $2 \pi n$, the corresponding net rotation of the tangent vector around $\Gamma$, obeys the rule $2 \pi+v=2 \pi n$, where $v$ is the variation of $\arg \left(F^{\prime}(z)\right)$ as $z$ traces $\gamma$.

But $F^{\prime}(z)=S(z) f(z)$, so it follows that $v=2 \pi(W(\gamma, S)+W(\gamma, f))$, where $W(\gamma, h)$ denotes the winding number about the origin of a function $h$, defined on $\gamma$, as $\gamma$ is traced counterclockwise.

I.e.,

$$
1+W(\gamma, S)+W(\gamma, f)=n,
$$

for some integer $n$. Now if $\gamma$ is a sufficiently small deformation of $\partial D$, it will be the case that $1+W(\gamma, S)=0$, since $W(\partial D, \bar{z})=-1$, and $W(\gamma, S)$ is integer-valued and varies continuously with $\gamma$. Thus, if $n$ is not equal to zero, we conclude from (2) that $W(\gamma, f)$ is not zero. This implies that the curve $f(\gamma)$ must circle the origin at least once, and so must certainly cut the real and imaginary axes. In particular, $u$ and $v$ must both have zeros on $\gamma$, so that in this case Property $\mathrm{P}$, rather than the weaker Property $\mathrm{Q}$, is established.

There remains the possibility that $n=0$, and we must show that in this case Property $\mathrm{Q}$ is valid. (Interestingly, it is easy to show that if $D=\Delta$, it is impossible for $n$ to be zero.)

In order to treat the case in which $n=0$, note that in this case $\Gamma$ must contain a simple loop that is smooth except for a single corner, and that is the image under $F$ of a subarc $A$ of $\gamma$. Furthermore, for such a loop the net rotation of the tangent vector is $\geq \pi$ in absolute value. Additionally, the counterpart of formula (2) is valid, so that

$$
\left|(2 \pi)^{-1} \theta(A)+W(A, S)+W(A, f)\right| \geq \frac{1}{2},
$$

where $\theta(A)$ is the net rotation of the tangent vector to $\gamma$ as $z$ moves along $A$. Now for the unit circle, $S(z)=z^{-1}$, so that if $\gamma$ were the unit circle, it would be the case that $(2 \pi)^{-1} \theta(A)+W(A, S)=0$. If $\gamma$ is $C^{2}$-close enough to the unit 
circle, it will therefore certainly be the case that $\left|(2 \pi)^{-1} \theta(A)+W(A, S)\right|<\frac{1}{4}$, so the curve $f(A)$ has a net winding number about the origin of at least $\frac{1}{4}$, which implies Property Q, since $f(A)$ must then cut either the real or the imaginary axis.

Remark. Recently Harold S. Shapiro has informed me that after reading a preprint of this note, he and Björn Gustafsson have begun an investigation of the multidimensional version of Property $P$, and that during the course of this investigation, Gustafsson, using techniques drawn from the study of free boundary problems, quadrature domains, and potential-theory, has in the 2dimensional case succeeded in demonstrating the equivalence of Property $\mathbf{P}$ with a quadrature property, and shown that such a quadrature property holds for compact domains having analytic boundary. Among other things, this implies that the last theorem is true with Property Q replaced by Property $\mathrm{P}$.

\section{REFERENCES}

1. Phillip J. Davis, Double integrals expressed as single integrals or interpolatory functionals, J. Approx. Theory 5 (1972), 276-307.

2. The Schwarz function and its applications, Carus Math. Monographs, no. 17, Math. Assoc. America, Washington, D.C., 1974.

3. Bernard Epstein, On the mean-value property of harmonic functions, Proc. Amer. Math. Soc. 13 (1962), 830.

4. G. Mittag-Leffler, Sur la représentation analytique d'une branche uniforme d'une fonction monogène, Acta Math. 29 (1905), 101-181.

5. Makoto Sakai, Quadrature domains, Lecture Notes in Math., vol. 934, Springer-Verlag, New York, 1982.

Department of Mathematics, CUNy Graduate Center, 33 West 42 Street, New York, NEW YORK 10036

E-mail address: trigc@cunyvm.bitnet 